\title{
Age-related differences in working memory performance in a 2-back task
}

\section{Nele Wild-Wall*, Michael Falkenstein and Patrick D. Gajewski}

Leibniz Research Centre for Working Environment and Human Factors, University of Dortmund (IfADo), Dortmund, Germany

\section{Edited by:}

Shulan Hsieh, National Cheng Kung

University, Taiwan

Reviewed by:

Nicola K. Ferdinand, Saarland

University, Germany

Yang Jiang, University of Kentucky, USA

${ }^{*}$ Correspondence:

Nele Wild-Wall, Leibniz Research Centre for Working Environment and Human Factors (IfADo), Ardeystr. 67, D-44139 Dortmund, Germany.

e-mail: wild-wall@ifado.de
The present study aimed to elucidate the neuro-cognitive processes underlying age-related differences in working memory. Young and middle-aged participants performed a two-choice task with low and a 2-back task with high working memory load. The P300, an event-related potential reflecting controlled stimulus-response processing in working memory, and the underlying neuronal sources of expected age-related differences were analyzed using sLORETA. Response speed was generally slower for the middle-aged than the young group. Under low working memory load the middle-aged participants traded speed for accuracy. The middle-aged were less efficient in the 2-back task as they responded slower while the error rates did not differ for groups. An age-related decline of the P300 amplitude and characteristic topographical differences were especially evident in the 2-back task. A more detailed analysis of the P300 in non-target trials revealed that amplitudes in the young but not middle-aged group differentiate between correctly detected vs. missed targets in the following trial. For these trials, source analysis revealed higher activation for the young vs. middle-aged group in brain areas which support working memory processes. The relationship between P300 and overt performance was validated by significant correlations. To sum up, under high working memory load the young group showed an increased neuronal activity before a successful detected target, while the middle-aged group showed the same neuronal pattern regardless of whether a subsequent target will be detected or missed. This stable memory trace before detected targets was reflected by a specific activation enhancement in brain areas which orchestrate maintenance, update, storage, and retrieval of information in working memory.

Keywords: aging, working memory, 2-back task, event-related potentials, P300

\section{INTRODUCTION}

Working memory can be seen as a mental buffer for temporal information retrieval from long-term memory, temporal storage of new information, and manipulation of this information in service of ongoing mental tasks. Thus, it is essential for mental performance and may subserve higher cognitive abilities like planning or reasoning (Hasher and Zacks, 1988). In one classical model by Baddeley working memory consists of at least three sub-processes, a central executive or attentional control system, a sketch pad for manipulation of information, and a storage and rehearsal component (Baddeley, 1992). Neuroimaging data indicate further sub-processes like manipulation or retrieval of information (Smith et al., 1998), which can be roughly integrated in the three sub-processes of Baddeley (1992).

It is widely accepted that working memory processes are supported at least by frontal and parietal brain areas (Missonnier et al., 2003; Chatham et al., 2011). Insight from neuroimaging studies suggest a differentiation of specific working memory functions in the respective brain areas: maintenance and rehearsal of information may be rather supported by the ventro-lateral frontal cortex (Fletcher and Henson, 2001) whereas manipulation of information in working memory is related to the dorsolateral frontal cortex (Smith et al., 1998; Fletcher and Henson, 2001) but possibly also to the superior parietal cortex (Koenigs et al., 2009). Parietal brain areas as, e.g., the intraparietal sulcus and posterior parietal cortex are rather thought to perform serial order representation (Marshuetz et al., 2006) as well as short-term recall of old information and the short-term storage of new information (Chatham et al., 2011).

\section{WORKING MEMORY AND AGE-RELATED CHANGES}

A decline in working memory is well documented with increasing age (Hasher and Zacks, 1988; Babcock and Salthouse, 1990; Li et al., 2001). Such decline has been shown with different paradigms like the delayed recognition task (Gazzaley et al., 2005) or the so called n-back task where a currently presented stimulus should be compared with previously presented stimuli (Nyberg et al., 2009; Schmiedek et al., 2009).

An age-related degradation of the neural substrate is pronounced in the frontal lobe (West, 1996; Raz et al., 1998) and may be related to the decline in working memory performance. However, the mechanisms of the age-related decline in working memory performance are not yet fully understood. A widely accepted hypothesis is an age-related deficit in filtering or suppressing irrelevant information which may in turn strain the limited capacity of working memory and reduce performance (Hasher and Zacks, 1988; Gazzaley et al., 2005). Related to this, it is well possible that the manipulation of information in working memory, a subprocess which is supported by frontal lobe brain areas (Fletcher and Henson, 2001), may be deficient with increasing age (Dobbs 
and Rule, 1989). It is less clear if age-related differences in processes which are supported by parietal areas may also contribute to agerelated differences in working memory performance. For example, Missonnier et al. (2004) discussed an inefficient recruitment of parietal neural resources to explain an age-related decline in working memory performance.

Against the background of the age-related frontal lobe degradation one may rather expect reduced activation of frontal lobe brain areas during high working memory load. However, several studies reported even increased activation of bilateral frontal lobe areas in older vs. young participants during working memory tasks as measured with neuroimaging methods (Reuter-Lorenz et al., 2000; Mattay et al., 2006). As such bilateral vs. unilateral frontal lobe activation in older participants was rather found for good vs. bad performers, it is usually interpreted as a compensatory mechanism (Reuter-Lorenz et al., 2001).

\section{THE PRESENT STUDY}

Although frontal lobe changes already start after the third decade (Salat et al., 1999), it is unclear when these neural changes have an impact on functional processes and become evident in overt performance, e.g., in tasks with high working memory demands. Therefore, in the present study we aimed to investigate if a functional decline in working memory can be already observed in middle-aged participants (i.e., between 40 and 65). This bears practical relevance because middle-aged participants are normally still active in working life. To this aim, we recruited young and middle-aged participants. They had to perform two tasks with low and high working memory load: a simple two-choice task and a 2-back task which induces a high working memory load. Here, the participants were asked to press one button to a stimulus if it corresponded to the stimulus in the last but one trial, requiring a continuous update of the memory content and comparing it with the stored one. During task performance the electroencephalogram (EEG) was recorded in order to calculate event-related potentials (ERPs) and more specifically the P300 as a measure of attentional and working memory processes (Polich, 2007) and to assess the source of potential differences in the P300 using sLORETA (Pascual-Marqui, 2002). According to the view of Polich (2007) the P300 (as a general labeling of this potential ${ }^{1}$ ) may be divided at least into two subcomponents, the more frontally distributed P3a and the more parietal distributed $\mathrm{P} 3 \mathrm{~b}$. The $\mathrm{P} 3 \mathrm{a}$ is thought to reflect the processing of novel stimuli as well as attentional and working memory processes and is supported by frontal brain areas. The P3b may reflect memory-related storage operations which are engaged by frontal attention and working memory processes and which are supported by temporal-parietal brain areas (Polich, 2007). The P3b was found to be generally reduced with increasing age (Friedman et al., 1997). In addition, the potential comprises a more frontal topography in older participants which was interpreted as the involvement of frontal brain areas for stimuli which should have been already well encoded (Friedman et al., 1997).

${ }^{1}$ Subsequently, we use the notation P3a whenever an effect is focused on more frontal electrode sites, we use P3b whenever the effect is focused on more parietal electrodes. We use the general notation P300 whenever the effect does not have any specific focus on the sagittal plane.
We expect generally slower response times (RT) in the middle-aged compared to the young participants (Wild-Wall and Falkenstein, 2010). In addition, we expect especially lower performance for the middle-aged participants under high working memory load, i.e., in the 2-back compared to the control task. This decline in performance may be also reflected in a lower amplitude of the P3b which should be especially pronounced in the 2-back task. As the 2-back task measures an online updating of ongoing events and the matching with the recently stored event, comparable processes may be involved in target as well as in non-target trials. In fact, the brain activity of non-target trials immediately preceding target trials may indicate more or less efficient online control and matching processes and thus, may be related to the success of detecting the following target. Therefore, we also examined the P300 in the non-target trials which directly preceded detected or missed targets in the 2-back task. We expect age-related differences in the brain activity to be apparent also in these non-target trials preceding targets. Possibly, the age-related differences may depend on the correctness of the following target trial.

Depending on the scalp focus of possible age-related differences in the P300 amplitude it may be possible to pinpoint specific sub-processes which may be compromised in the middle-aged participants under high working memory load. If also the frontally distributed P3a is decreased for the middle-aged vs. young participants, this may point to a decline in sub-processes of working memory which are supported by the frontal lobe. If, on the other hand, the P3a may be even increased in the middle-aged vs. young participants, this may suggest compensatory mechanisms as was, e.g., discussed by Reuter-Lorenz et al. (2001).

\section{MATERIALS AND METHODS PARTICIPANTS}

For the study, 90 healthy male participants were recruited from two age groups, two of them were left-handed. In the middle-aged group the participants had a mean age of 51 years $(48-59$ years; $n=46)$. The young group ( $n=44$ ) had a mean age of 22 years ( $19-31$ years). All participants had normal or corrected-to-normal vision and gave informed consent prior to participation of the study. They received a payment for their participation.

\section{DESIGN AND TASKS}

The stimulus material of both tasks consisted of 25 capital letters from the alphabet which were presented in white on a dark gray computer screen. The size of the stimuli was $12 \mathrm{~mm} \times 18 \mathrm{~mm}$ (width and height) which corresponds to a viewing angle of $1.2^{\circ}$ and $1.7^{\circ}$ at a viewing distance of $60 \mathrm{~cm}$. In the two-choice (or 0-back) task 196 and in the 2-back task 388 trials were presented consisting of $20 \%$ target letters and $80 \%$ non-target letters. In the two-choice task the participants were instructed to press the left button for the non-target letters and the right button for the target letters X. In the 2-back task the participants were instructed to press the right button if the current letter matches the letter which was presented two trials before (target) and the left button whenever the letters do not match (non-target). 


\section{ERP RECORDINGS}

The EEG was recorded continuously from 32 active scalp electrodes according to the extended 10-20 system (Pivik et al., 1993) which were mounted into an elastic cap. The montage included eight midline sites and 12 sites on each hemisphere. Alongside the EOG was recorded from electrodes placed above and below both eyes (vEOG) and next to the outer canti (hEOG). The signals were sampled and amplified with $2048 \mathrm{~Hz}$. The amplifier bandpass was 0.01-140 Hz. Electrode impedance was kept below $10 \mathrm{k} \Omega$.

\section{DATA ANALYSIS}

In both tasks, all trials with incorrect responses and trials which were faster than $100 \mathrm{~ms}$ or slower than $2000 \mathrm{~ms}$ were excluded from analysis of the RT and ERPs. For each task and participant, mean RTs, and error rates were calculated for the target as well as the non-target conditions. RTs and error rates were subjected to ANOVAs including the within-factor trial-type (target, non-target) and the betweenfactor group (middle-aged, young). In addition, a performance index - the inverse efficiency (IE) was calculated for each condition and participant by dividing the RT by the rate of correct responses (Townsend and Ashby, 1983). The IE (expressed in ms) provides a combined measure of RT and errors and therefore accounts for a possible tradeoff between speed and accuracy especially in the middle-aged group. The inverse efficiencies (IE) of both tasks were also subjected to ANOVAs including the factors trial-type and group.

Offline, the EEG was downsampled to $1000 \mathrm{~Hz}$ and cut into epochs of $1400 \mathrm{~ms}$ length corresponding to $100 \mathrm{~ms}$ before and $1300 \mathrm{~ms}$ after stimulus onset. Eye movements were corrected using the method described by Gratton et al. (1983). Epochs with absolute amplitudes above $200 \mu \mathrm{V}$ and with voltage steps of more than $80 \mu \mathrm{V}$ between sampling points were rejected as artifacts. For each task, participant and electrode the epochs including correct responses were averaged according to trial-type (target vs. non-target) with a pre-stimulus baseline of $100 \mathrm{~ms}$. Additionally, the epochs of correct non-target trials in the 2-back task which directly preceded target trials were sorted according to the correctness of the following target trial and averaged for each participant and electrode. In other words, the P300 in trial $n-1$ was analyzed as a function of correct or incorrect targets in trial $\mathrm{n}$.

The amplitude of the $\mathrm{P} 300$ potential was quantified at selected electrodes (FC3, FCz, FC4, C3, Cz, C4, CP3, CPz, CP4, P3, Pz, P4) in the average ERPs for each task, participant and condition as the maximal peak in the time interval from 300 to $600 \mathrm{~ms}$ after stimulus onset. For each task the P300 was first analyzed with an ANOVA including the within-factors trial-type (target, non-target), sagittal row (FC, C, CP, P), laterality (left, midline, right electrodes) and the between-factor group (middle-aged, young).

Only for the 2-back task a more confined analysis of the non-target trials directly preceding targets was conducted including the withinfactors sagittal row, laterality, correctness of the following target (following target correct or incorrect), and the between-factor group. The underlying sources of possible amplitude differences between conditions or groups were further analyzed with sLORETA (PascualMarqui, 2002). This program estimates the sources of activation on the basis of standardized current density at each of 6239 voxels in the gray matter of the MNI-reference brain with a spatial resolution of $5 \mathrm{~mm}$. The calculation is based upon a linear weighted sum of the scalp electric potentials with the assumption that neighboring voxels have a maximal similar electrical activity. The voxel-based sLORETA images were first computed for each individual averaged ERP in the interval from 350 to $450 \mathrm{~ms}$ surrounding about the $\mathrm{P} 300$ peak in the non-target trials for both groups. Then, two comparisons were calculated which were based on the results of the previous analysis of the P300 in the non-target trials preceding the targets: (a) the sLORETA images of the non-target condition before correct targets were statistically compared between groups and (b) the sLORETA images of the non-target condition before correct and before missed targets. The sLORETA voxelwise randomization test (5000 permutations) which is based on statistical non-parametric mapping (SnPM) and implemented in sLORETA was used for comparison. For each analysis, one single test was carried out for an average of all time frames in the critical interval. The tests were corrected for multiple comparisons (Holmes et al., 1996).

\section{RESULTS}

\section{PERFORMANCE}

\section{Low working memory load (two-choice task)}

The performance data of the task are summarized in Table 1. The RTs were faster to the non-targets $(336 \mathrm{~ms})$ than to the targets $[398 \mathrm{~ms} ; F(1,88)=279.3, p<0.001]$. In addition, the middle-aged participants showed slower RTs than the young group [390 vs. $344 \mathrm{~ms}: F(1,88)=25.5, p<0.001]$. Error rates to targets (misses: $9.1 \%$ ) were higher than to non-targets [false alarms: $0.7 \% ; F(1,88)=171.5, p<0.001]$. In addition, the middle-aged participants committed generally less errors $(3.1 \%)$ than the young group $[6.7 \% ; F(1,88)=27.6, p<0.001]$. The two main effects were qualified by an interaction of trial-type and group $[F(1,88)=22.6$, $p<0.001]$. However, post hoc tests revealed that the middle-aged participants showed lower error rates for both, misses [5.8 vs. $12.6 \% ; F(1,88)=25.6, p<0.001]$ as well as for false alarms to nontargets $[0.4$ vs. $1.0 \% ; F(1,88)=8.4, p<0.01]$ than the young group.

The IE were higher (i.e., lower performance) for the targets $(442 \mathrm{~ms})$ than for the non-targets $[339 \mathrm{~ms} ; F(1,88)=274.7$, $p<0.001]$. A main effect of group $[F(1,88)=6.9, p=0.01]$ was qualified by an interaction with trial-type $[F(1,88)=7.8, p<0.01]$ : the middle-aged vs. young participants showed lower performance (i.e., higher IE) for the non-target stimuli [361 vs. $315 \mathrm{~ms}$ : $F(1,88)=30.2, p<0.001]$ but comparable performance for the target condition ( 447 vs. $436 \mathrm{~ms}: F<1$ ).

\section{High working memory load (2-back task)}

Analysis of the RTs in the 2-back task (see Table $\mathbf{1}$ for a summary of performance) yielded two significant main effects with slower RTs for targets than for non-targets [545 vs. $395 \mathrm{~ms} ; F(1,88)=151.8$, $p<0.001$ ] as well as for the middle-aged than for the young group [ 463 vs. $387 \mathrm{~ms} ; F(1,88)=24.9, p<0.001]$. For error rates there was only a main effect of trial-type with more errors committed in the target condition (31\%) than in the non-target condition $[2.7 \%$; $F(1,88)=333.1, p<0.001]$. No other effect reached significance $(F s<1)$.

The IE in the 2-back task was higher (i.e., lower performance) in the target $(705 \mathrm{~ms})$ than in the non-target condition [407 ms; $F(1,88)=146.4, p<0.001]$ as well as for the middle-aged group (615 ms) than for the young group $[495 \mathrm{~ms} ; F(1,88)=15.8$, $p<0.001]$. The interaction did not reach significance. 
Table 1 | Performance data of the middle-aged and the young group in the two-choice task (upper panel) and the 2-back task (lower panel).

\begin{tabular}{|c|c|c|c|}
\hline & Middle-aged group & Young group & \\
\hline \multicolumn{4}{|l|}{ TWO-CHOICE TASK } \\
\hline Response times - overall (ms) & 390 & 344 & $p<0.001$ \\
\hline Response times - targets (ms) & $420(52.9)$ & $376(51.7)$ & $\mathrm{n} . \mathrm{s} / \mathrm{p}<0.10$ \\
\hline Response times - non-targets (ms) & $360(43.2)$ & $312(35.6)$ & $\mathrm{n} . \mathrm{s} / \mathrm{p}<0.10$ \\
\hline Error\% - overall & 3,1 & 6,7 & $p<0.001$ \\
\hline Inverse efficiencies - overall (ms) & 404 & 375 & $p<0.01$ \\
\hline Inverse efficiencies - targets (ms) & $447(57.8)$ & 436 (87.4) & $\mathrm{n} . \mathrm{s} / \mathrm{p}<0.10$ \\
\hline Inverse efficiencies - non-targets (ms) & $361(42.5)$ & $315(36.2)$ & $p<0.001$ \\
\hline \multicolumn{4}{|l|}{ 2-BACK TASK } \\
\hline Response times - overall (ms) & 463 & 387 & $p<0.001$ \\
\hline Error\% - non-targets & $2.9(2.0)$ & $2.4(2.2)$ & $\mathrm{n} . \mathrm{s} / \mathrm{p}<0.10$ \\
\hline Inverse efficiencies - overall (ms) & 615 & 495 & $p<0.001$ \\
\hline Inverse efficiencies - targets (ms) & $785(290.0)$ & $623(199.5)$ & $p<0.001$ \\
\hline Inverse efficiencies - non-targets (ms) & $446(78.1)$ & $367(69.6)$ & $p<0.001$ \\
\hline
\end{tabular}

The SDs are given in parentheses. The significance levels of group differences are indicated in the last column.

\section{ERP RESULTS (P300)}

\section{Low working memory load (two-choice task)}

The ANOVA (see Figure 1) revealed a main effect of sagittal row $[F(3,264)=45.5, p<0.001]$ with highest amplitudes of the P300 at centro-parietal $(15.8 \mu \mathrm{V})$ when compared to fronto-central, central, or parietal electrodes $(13.4 / 15.2 / 15.0 \mu \mathrm{V})$ with the central row being also significantly higher in amplitude than the fronto-central or parietal row [all $F s(1,88)>17.3$, all $p s<0.001$ ]. In addition, the P300 showed a midline and right topography [main effect of laterality: $F(2,176)=126.6, p<0.001]$ with highest amplitudes at midline electrodes $(16.4 \mu \mathrm{V})$, lower amplitudes at the right $(15.1 \mu \mathrm{V})$ and even lower ones at the left side $\left[13.3 \mu \mathrm{V}\right.$; all $F_{s}(1,88)>49.8$, both $p s<0.001]$. The P300 amplitude to targets $(18.7 \mu \mathrm{V})$ was higher than to non-targets [main effect of trial-type; $11.0 \mu \mathrm{V}$; $F(1,88)=378.6, p<0.001]$.

There were two two-way interactions of sagittal row $\times$ group $[F(3,264)=11.8, p<0.001]$ and of target condition $\times$ sagittal row $[F(3,264)=27.8, p<0.001]$ which were qualified by a three-way interaction of trial-type $\times$ sagittal row $\times$ group $[F(3,264)=5.8$, $p<0.01]$. For the non-target condition the P300 amplitude was comparable between groups at all sagittal rows. In contrast, for the target condition, the P300 amplitude was higher in the young compared to the middle-aged group at the centro-parietal (21.5 vs. $18.5 \mu \mathrm{V})$ and the parietal row $[20.9$ vs. $17.4 \mu \mathrm{V}$; both $F s(1,88)>5.2$, both $p s<0.05$ ]. In addition, for the young group the P300 at the fronto-central row was generally smaller than at central to parietal electrode sites [all $F s(1,43)>14.7$, all $p s<0.001]$. For the middleaged participants the P300 showed a central and centro-parietal maximum and smaller amplitudes at fronto-central as well as parietal electrode sites [all $\mathrm{Fs}(1,45)>11.8$, all $p s<0.01]$.
In summary, in the task with low working memory load, the main age-related P300 result in a selective parietal attenuation in the less frequent target trials for the middle-aged compared to the young group.

\section{High working memory load (2-back task)}

The P300 in the 2-back task (Figure 2) was generally higher for targets $(14.7 \mu \mathrm{V})$ than non-targets $[8.1 \mu \mathrm{V} ; F(1,88)=201.7$, $p<0.001]$ as well as for the younger $(12.6 \mu \mathrm{V})$ than for the middle-aged group $[10.3 \mu \mathrm{V} ; F(1,88)=5.2, p<0.05]$. The main effect of sagittal row was significant $[F(3,264)=28.6, p<0.001]$ : lowest P300 amplitudes were found at the fronto-central row $(10.4 \mu \mathrm{V})$, highest amplitudes at the centro-parietal row $(12.1 \mu \mathrm{V})$, and intermediate amplitudes at the central and parietal rows which did not differ significantly [11.7 and $11.5 \mu \mathrm{V} ; F<1$; all other $F s(1,88)>11.8$, all other $p s<0.001]$. The significant main effect of sagittal row was further qualified by an interaction with the factor group $[F(3,264)=18.2, p<0.001]$ : the groups did not differ in P300 amplitude at the fronto-central $(F<1)$ and central row $[F(1,88)=3.5, p=0.065]$ but amplitudes were significantly higher for the young vs. middle-aged group at the centro-parietal $(13.5$ vs. $10.7 \mu \mathrm{V})$ and parietal row [13.3 vs. $9.8 \mu \mathrm{V}$; both $F s(1,88)>7.9, p s<0.01]$. In addition, whereas the middle-aged group showed a central to centro-parietal $(10.6 / 10.7 \mu \mathrm{V})$ maximum of the P300 with lower amplitudes at fronto-central and parietal sites [10.0/9.8 $\mu \mathrm{V}$; all $F s(1,45)>8.0$, all $p s<0.01]$, the young group showed a clear centro-parietal to parietal maximum of the P300 $(13.5 / 13.3 \mu \mathrm{V})$ with lower amplitudes at the fronto-central and central row $\left[10.8 / 12.7 \mu \mathrm{V}\right.$; all $\mathrm{Fs}_{\mathrm{s}}(1,43)>18.3$, all $p s<0.001]$. 

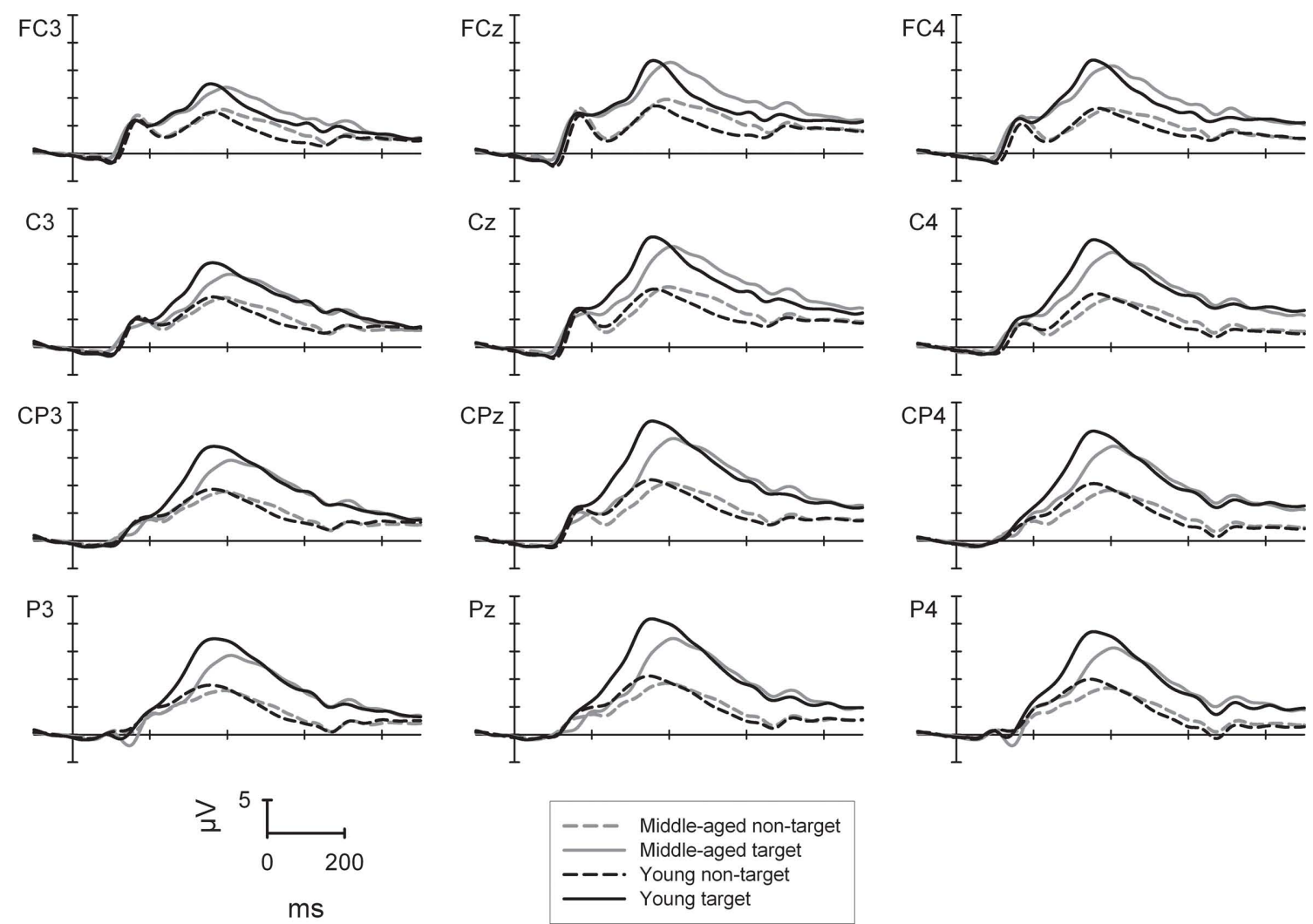

FIGURE 1 | P300 potential at selected electrode sites for the two-choice task (low working memory load) separately for the young (black lines) and middle-aged group (gray lines) as well to target (solid lines) and non-target stimuli (dashed lines).

The interaction between the factors trial-type and group was significant $[F(1,88)=25.2, p<0.001]$ : whereas the amplitude differed significantly between groups for the target condition [young: $17.0 \mu \mathrm{V}$, middle-aged: $12.4 \mu \mathrm{V} ; F(1,88)=11.3, p<0.001]$, the P300 amplitudes were comparable between groups in the non-target condition (both groups $8.2 \mu \mathrm{V} ; F<1$ ).

The P300 showed generally a central to right lateralized topography $[F(2,176)=90.5, p<0.001]$ with highest amplitudes at the midline $(12.3 \mu \mathrm{V})$, lower ones at the right side $(11.6 \mu \mathrm{V})$ and lowest amplitudes at the left side $[10.3 \mu \mathrm{V}$; all $F s(1,88)>22.3$, all $p s<0.001]$. Importantly, this main effect of laterality was qualified by a threeway interaction of trial-type $\times$ laterality $\times$ group $[F(2,176)=5.1$, $p<0.01]$. For young participants the $\mathrm{P} 300$ to non-targets was lower at left electrodes $(7.6 \mu \mathrm{V})$ when compared to central and right electrodes [ 8.3 and $8.5 \mu \mathrm{V}$; both $F s(1,43)>13.3$, both $p s<0.001$ ]. For the middle-aged participants, however, lower P300 amplitudes were found at left and right electrodes $(8.1$ and $7.7 \mu \mathrm{V})$ when compared to central electrodes [8.7 $\mu \mathrm{V}$; both $F \mathrm{~s}(1,45)>15.1$, both $p s<0.001]$. The P300 amplitude to targets showed a pattern left $<$ right $<$ central pattern for the young participants $[14.9<17.6<18.5 \mu \mathrm{V}$; all $F s(1,43)>11.2$, all $p s<0.002]$. This pattern was almost comparable for the middle-aged group: left $<$ central $=$ right $[10.7,13.5$ and $13.0 \mu \mathrm{V}$; both $\mathrm{Fs}(1,45)>69.7$, both $p s<0.001]$.

In summary, the frontal shift of the P300 was again observed in middle-aged participants. Similar to the data pattern in the twochoice task, the $\mathrm{P} 300$ was less positive in middle-aged than young participants in target but not in non-target trials. This effect in target trials, however, applies to the parietal as well as to the frontocentral P300. In addition and in contrast to the two-choice task, characteristic differences of the P300 topography between groups were evident also in non-target trials especially at right localized electrodes.

\section{Analysis of the non-target trials directly preceding target trials}

The more confined analysis of the P300 in non-target trials which directly preceded target trials (see Figure 3 ) showed a main effect of sagittal row $[F(3,264)=10.8, p<0.001]$ with higher $\mathrm{P} 300$ amplitudes at the central and centro-parietal row $(9.7$ and $9.9 \mu \mathrm{V})$ when compared to both, the fronto-central $(8.8 \mu \mathrm{V})$ and the parietal row [9.3 $\mu \mathrm{V}$; both $F s(1,88)>19.6$, both $p s<0.001]$. The main effect of sagittal row interacted significantly with group $[F(3,264)=14.9$, $p<0.001]$. Whereas for the young group the $\mathrm{P} 300$ showed a parietal maximum with higher amplitudes at the central, centro-parietal, and parietal rows $(9.6 / 10.2 / 9.9 \mu \mathrm{V})$ when compared to the frontocentral row $\left[8.2 \mu \mathrm{V}\right.$, all $F_{s}(1,43)>8.1$, all $\left.p s<0.007\right]$ the middleaged group showed higher amplitudes at the central row $(9.9 \mu \mathrm{V})$ compared to all other rows [9.4/9.5/8.6 $\mu \mathrm{V}$; all $F \mathrm{~s}(1,45)>5.5$, all ps $<0.023$ ].

An interaction of laterality $\times$ group $[F(2,176)=7.5, p<0.001]$ revealed that the $\mathrm{P} 300$ in the middle-aged group was higher at the midline $(10.0 \mu \mathrm{V})$ when compared to left or right lateralized electrodes [9.2/8.8 $\mu \mathrm{V}$; both $F s(1,45)>16.6$, both $p s<0.001]$. For the 

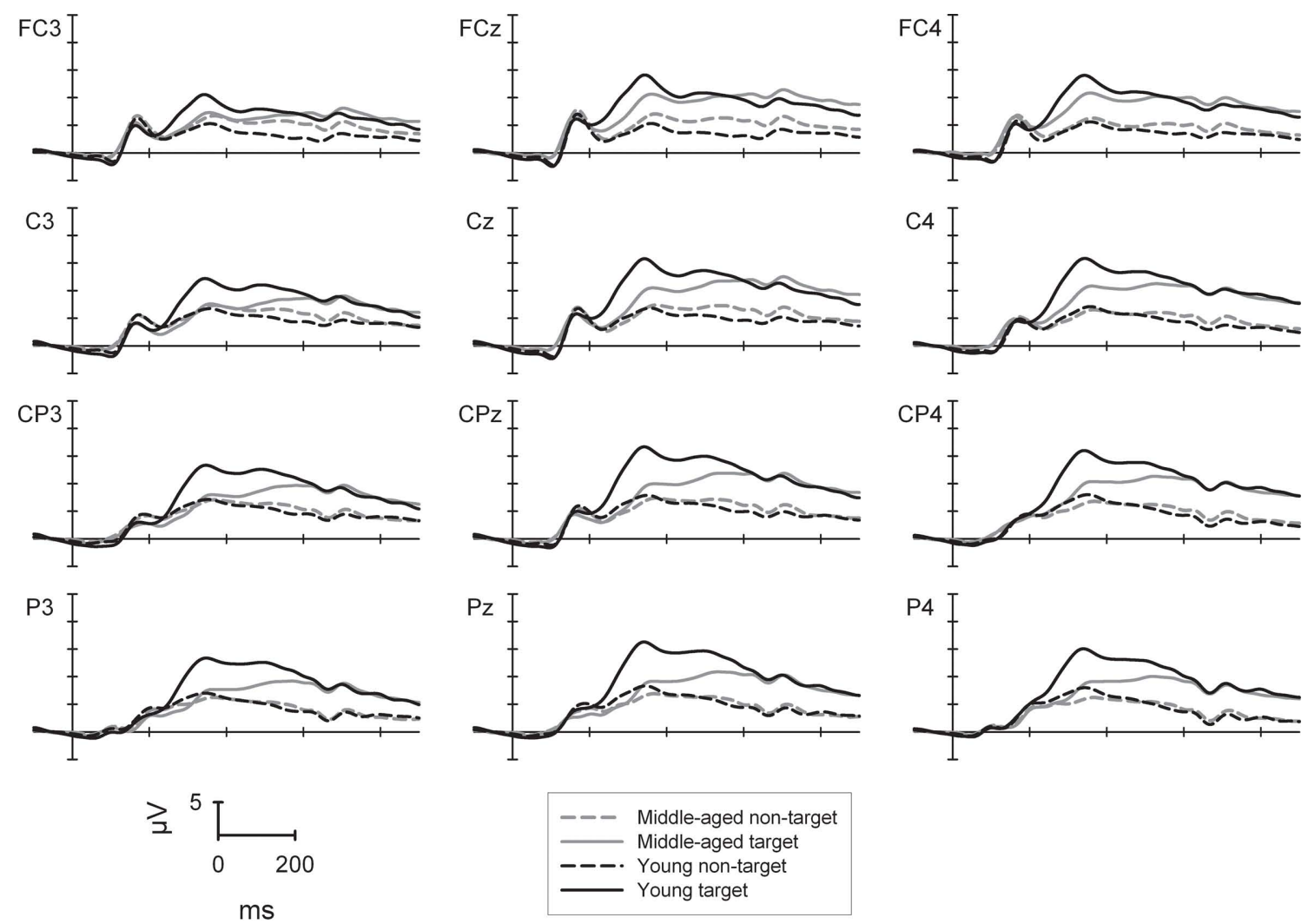

FIGURE 2 | P300 potential at selected electrode sites for the 2-back task (high working memory load) separately for the young (black lines) and middleaged group (gray lines) as well to target (solid lines) and non-target stimuli (dashed lines).

young group the P300 was lower at left electrodes $(8.8 \mu \mathrm{V})$ when compared to the midline and right electrodes $[10.0 / 9.6 \mu \mathrm{V}$; both $F s(1,43)>12.7$, both $p s<0.001]$. Importantly, a main effect of correctness $[F(1,88)=9.4, p<0.01]$ and a further interaction of correctness $\times$ group $[F(1,88)=10.6, p<0.01]$ was due to significantly higher P300 amplitudes for trials preceding correct vs. incorrect targets only for the young group [10.5 vs. $8.5 \mu \mathrm{V} ; F(1,43)=14.3$, $p<0.001]$ but not for the older group $(9.3$ vs. $9.4 \mu \mathrm{V} ; F<1)$.

There was a three-way interaction of laterality $\times$ correctness $\times$ group $[F(2,176)=7.9, p<0.001]$. This was mainly due to significantly higher P300 amplitudes for the young compared to the older group only in non-target trials preceding correct targets at right electrode positions [ 10.7 vs. $8.6 \mu \mathrm{V} ; F(1,88)=4.9, p<0.05$ ].

Exploratory correlations of the P300 amplitudes (at CP4) in non-target trials directly preceding correct targets with the IE to targets in the 2-back task revealed significant correlations for the middle-aged $(r=-0.33, p<0.05)$ as well as for the young group $(r=-0.43, p<0.01)$; in other words, the higher the $\mathrm{P} 300$ amplitude at right parietal sites the better the performance in the 2-back task (see Figure 4).

The sLORETA analysis of non-target trials preceding correct target trials revealed higher activation for the young compared to the older group in bilateral medial/inferior frontal areas, right middle frontal areas, right inferior parietal areas as well as in the cingulum (Figure 5; for statistics see Table 2). The analysis revealed a difference in the P300 amplitude for non-target trials before correctly vs. missed targets only for the young group when comparing the activation in these trials only for this group, there is significantly higher activation in the posterior cingulum and the right insula in trials preceding correct targets than in those nontarget trials preceding missed targets (Figure 6; for statistics see Table 2; Figure 3B).

In summary, in trials preceding a target stimulus, the P300 differentiates between detected and not-detected targets in the following trial in young but not in middle-aged participants. This pattern was supported by sLORETA that indicates higher activation in the posterior cingulum and the right insula for correctly detected than missing targets in young persons. Most importantly, the P300 in the non-target trials preceding a detected target was significantly attenuated for the middle-aged compared to the young group at right lateralized electrodes. This difference may be explained by lower activation of the middle-aged vs. young group in frontal and parietal brain areas which support working memory. Finally, there were significant correlations between the P300 amplitude and IE which suggest that the $\mathrm{P} 300$ is a reliable index of overt performance.

\section{DISCUSSION}

\section{PERFORMANCE AND GENERAL RESULTS}

In the present study we ask whether an age-related decline under high working memory load can be already observed in middle-aged participants. We additionally aimed to investigate the underlying processes of a possible decline. Furthermore, the present study complements 

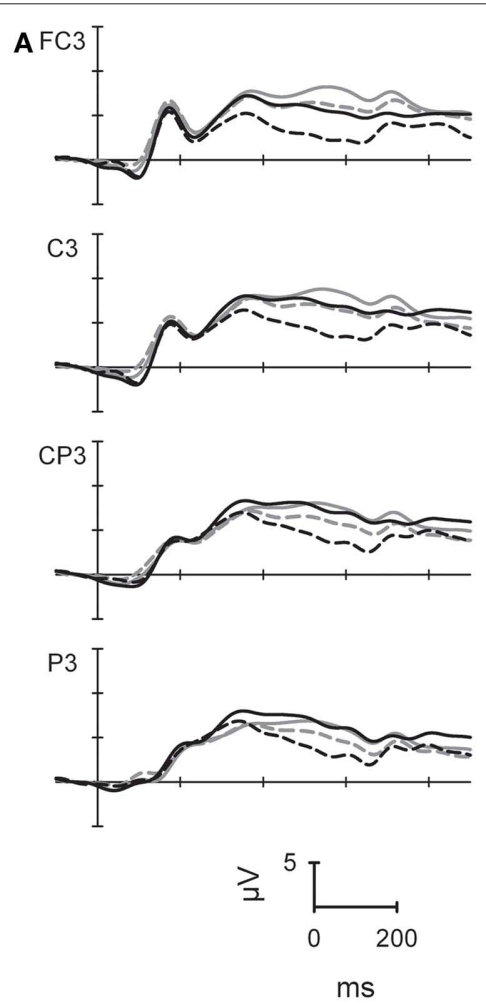
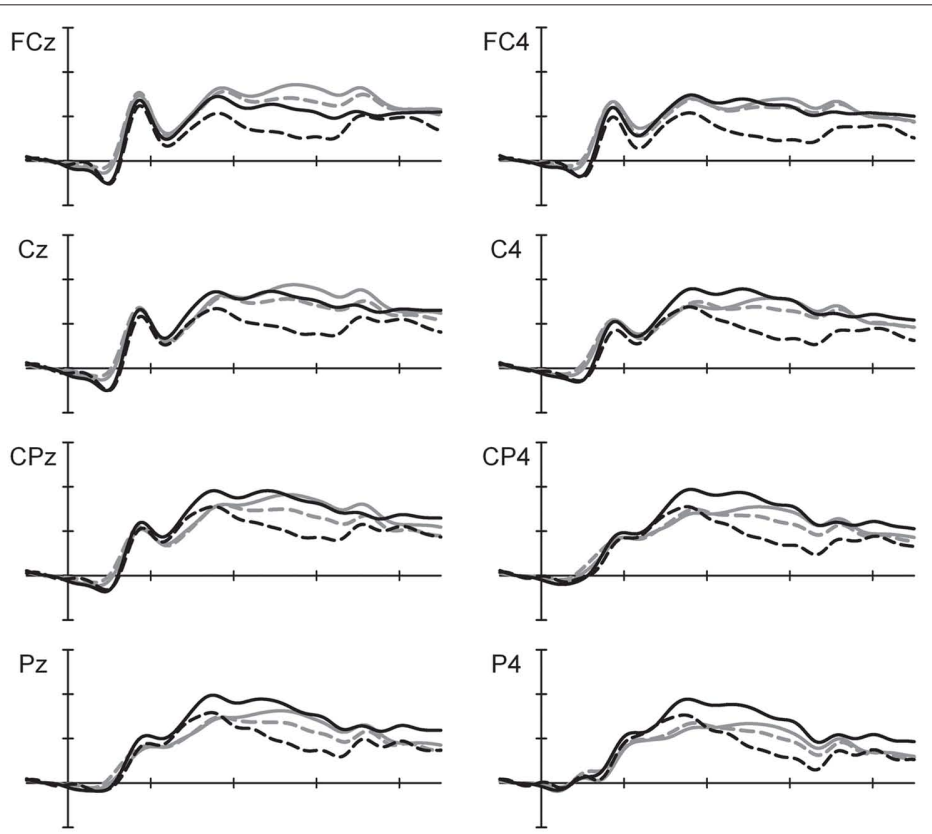

B

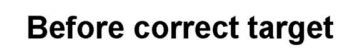

$$
\begin{aligned}
& \text { Middle-aged non-target before missed target } \\
& - \text { Middle-aged non-target before correct target } \\
& --- \text { Young non-target before missed target } \\
& - \text { Young non-target before correct target }
\end{aligned}
$$

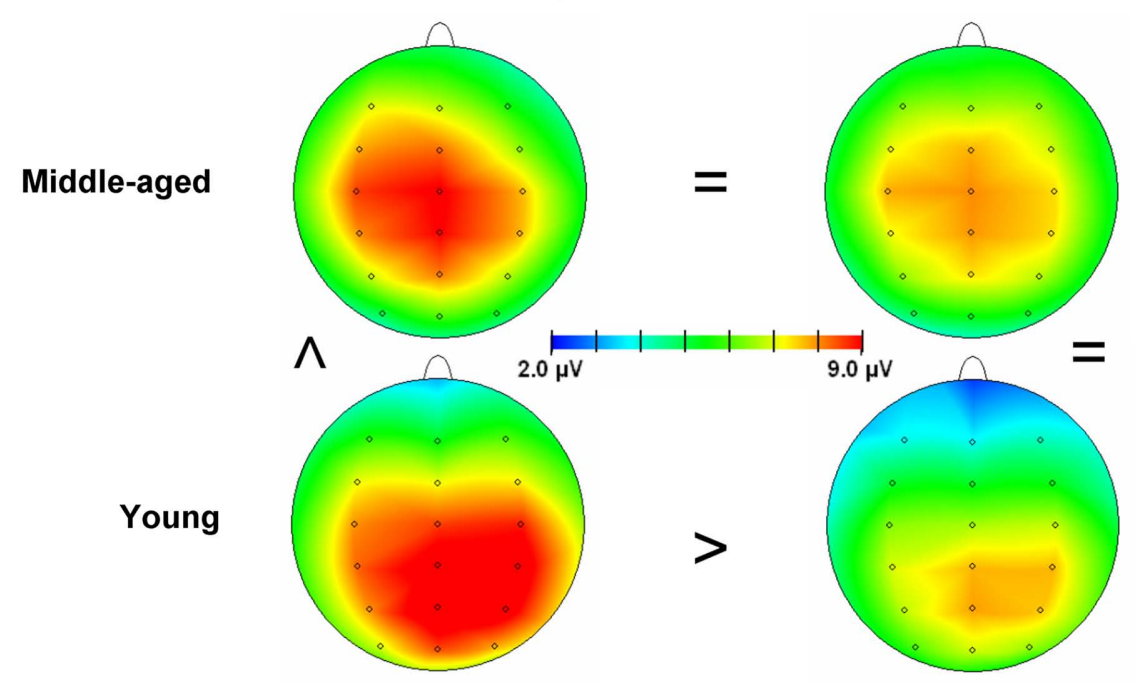

FIGURE 3 | (A) P300 in the 2-back task at selected electrodes for the middle-aged (gray lines) and young group (black lines) to correctly classified non-target stimuli which directly preceded correctly detected (solid lines) or missed targets (dashed lines) in the following trial. (B) Shows the maps of the
P300 at the time of the potentials highest peak separately for the groups and according to the correctness of the following target trial. The greater-than and the equal signs indicate the major amplitude differences between conditions and groups. other studies (Missonnier et al., 2003, 2004; Mattay et al., 2006; Nyberg et al., 2009) because we also examined the processes in non-target trials in relation to the performance in the target trials.

As expected, the middle-aged participants showed generally slower RTs than the young participants, reflecting the well known age-related slowing (Salthouse, 2000). However, error rates were lower for the middle-aged than the young participants in the easy two-choice task and similar in both groups in the 2-back task. Importantly, the analysis of the IE as an integrative index of performance showed no group effect, suggesting that the performance differences between groups in the easy two-choice task can be explained by a speed-accuracy tradeoff, that is, higher RTs and 


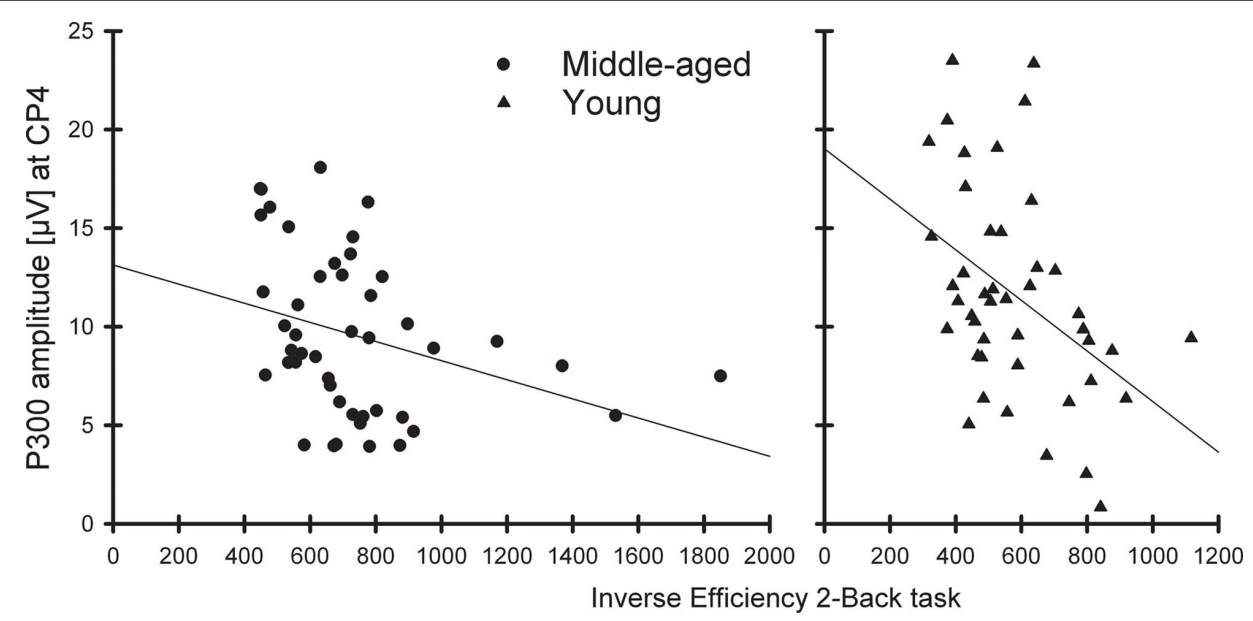

FIGURE 4 | Correlation between the P300 amplitude (CP4 electrode) to non-targets before correctly detected targets with the inverse efficiency to targets in the 2-back task separately for the middle-aged (left) and young group (right).
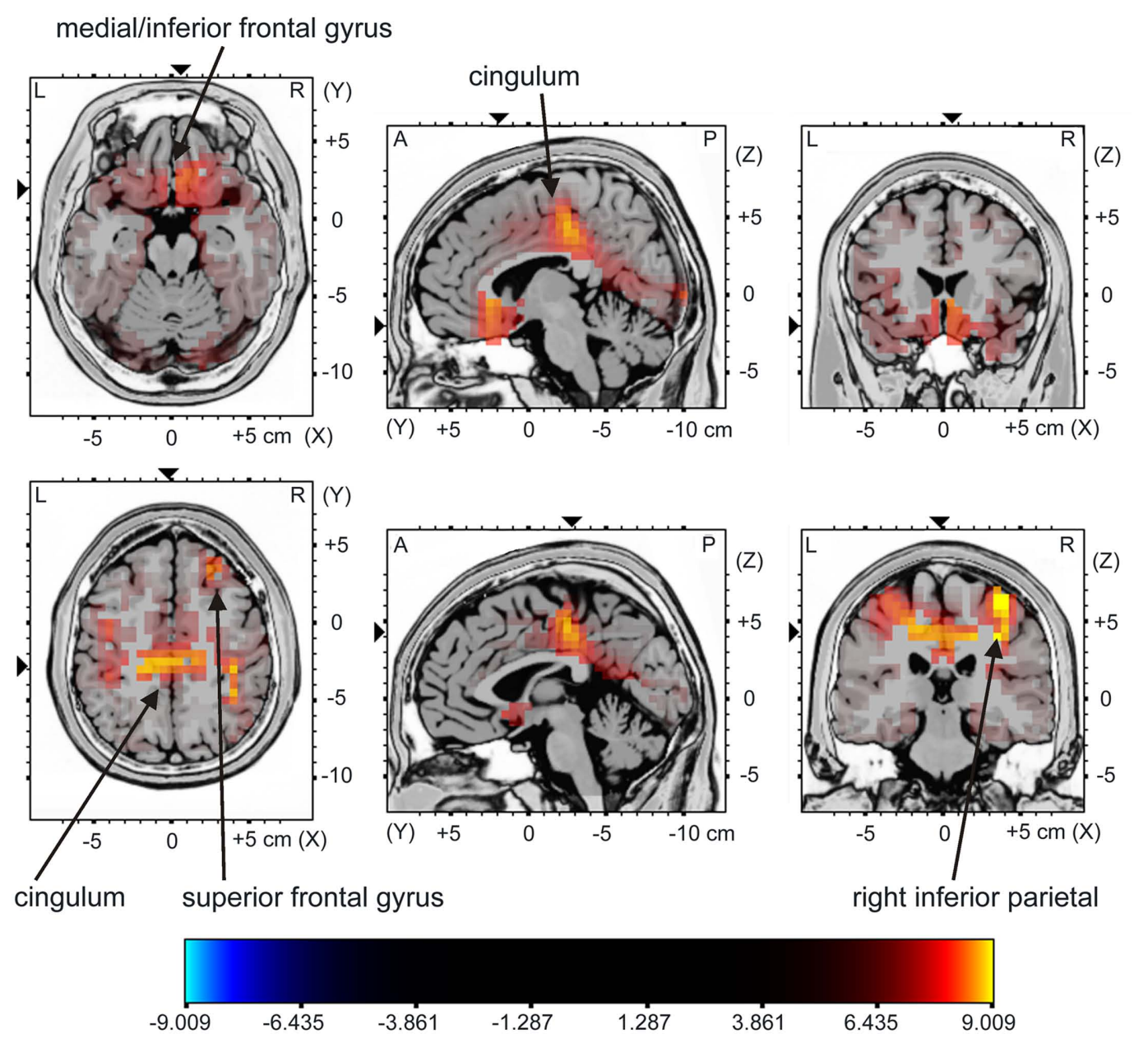

FIGURE 5 | sLORETA contrast of the P300 for young vs. middle-aged participants in non-target trials directly preceding correctly classified target stimuli as overlay on a reference brain. The red to yellow color represents voxels which show significantly higher activation for the young compared to the middle-aged group. For reasons of visualization, the upper panel shows sectional planes at $(x ; y ; z)=(-6 ; 19 ;-19)$ and the lower panel shows sectional planes at $(x ; y ; z)=(2 ;-30 ; 44)$. The main brain areas are labeled by arrows. 
Table 2 | Summary of the sLORETA results of the P300 to non-targets which directly preceded targets in the 2-back task.

\begin{tabular}{|c|c|c|c|c|c|}
\hline \multirow[t]{2}{*}{ BA } & \multirow[t]{2}{*}{ Location } & \multicolumn{3}{|c|}{ Coordinates } & \multirow{2}{*}{$\begin{array}{l}\begin{array}{l}\text { Critical } \\
t \text {-value }\end{array} \\
(p<0.01)\end{array}$} \\
\hline & & $x$ & $Y$ & $z$ & \\
\hline \multicolumn{6}{|c|}{ YOUNG VS. MIDDLE-AGED GROUP: ONLY PRECEDING CORRECT TARGETS } \\
\hline 10 & Right middle/superior frontal & $29 / 41$ & $42 / 52$ & $28 / 37$ & 3.77 \\
\hline 40 & Right inferior parietal & $30 / 50$ & $-30 /-53$ & $37 / 61$ & 3.77 \\
\hline \multicolumn{6}{|c|}{ PRECEDING CORRECT VS. PRECEDING MISSED TARGETS: ONLY YOUNG GROUP } \\
\hline 13 & Right insula & $39 / 42$ & $-3 /-17$ & $7 / 16$ & 3.9 \\
\hline $23 / 31$ & Posterior cingulum & $-6 / 7$ & $-29 /-45$ & $24 / 29$ & 3.9 \\
\hline
\end{tabular}

Only significant results at $p<0.01$ are reported. The Brodman areas (BA) are given in the first column.

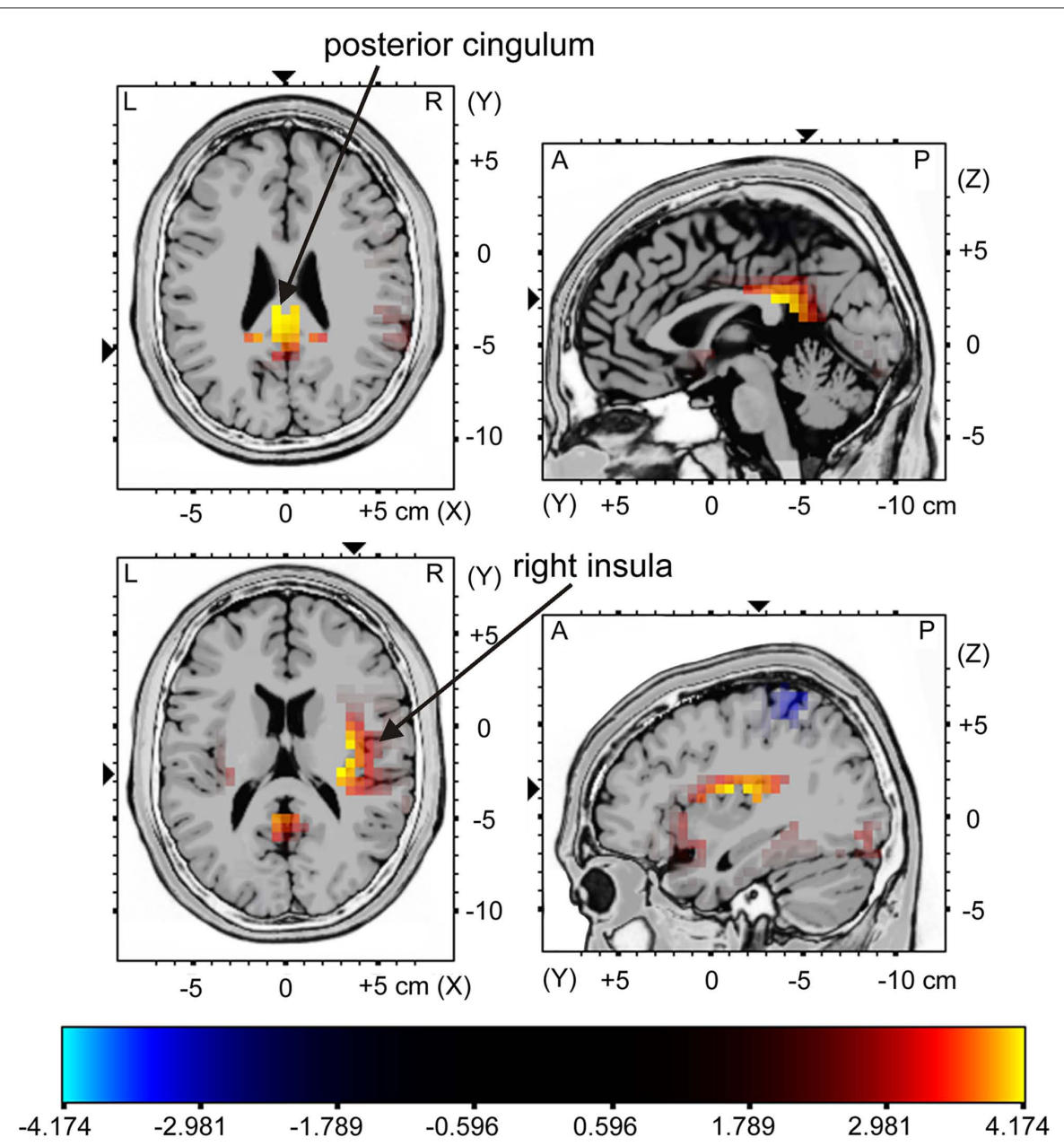

FIGURE 6 | sLORETA contrast of the P300 for the young participants in non-target trials directly preceding correctly classified target stimuli vs. missed target stimuli. The red to yellow color represents voxels which show higher activation for the trials preceding correctly classified targets vs. missed targets. For reasons of visualization, the upper panel shows sectional planes at $(x ; y ; z)=(1 ;-36 ; 24)$ and the lower panel shows sectional planes at $(x ; y ; z)=(37 ;-21 ; 16)$. The main brain areas are labeled by arrows. 
lower error rates in the middle-aged group and the reversed pattern in the young group. Hence in sum there is no performance deficit of the middle-aged group in the easy task. In contrast, a general performance decline was clearly evident for the middle-aged vs. young group in the 2-back task, i.e., under high working memory load. This is in line with other studies which also showed working memory decline with increasing age (Li et al., 2001; Missonnier et al., 2004; Gazzaley et al., 2005; Schmiedek et al., 2009). The present results extend these studies by showing that a decline in performance can be observed already in middle-aged participants in the fifth decade of age under high, but not under low, working memory load.

\section{NEUROPHYSIOLOGY OF AGE-RELATED WORKING MEMORY DIFFERENCES}

Analysis of the P300 revealed further insights into the neural underpinnings of the observed age-related decline. In both tasks the parietally pronounced $\mathrm{P} 3 \mathrm{~b}$ was generally increased to targets than to non-targets. This may be expected because the $\mathrm{P} 3 \mathrm{~b}$ reflects generally the allocation of processing resources to potentially relevant stimuli (Polich, 2007), that is, the target stimuli in both of the present tasks. Because in the two presented tasks the nontarget stimuli were also relevant for responding, another factor may have additionally contributed to the increased P300 for targets vs. non-targets. Especially the frontal sub-component of the potential (P3a) was found to be increased in amplitude to less frequent (like targets in the present study) as compared to frequent stimuli (Sutton et al., 1965; Polich, 2007). It is likely that both of these factors (relevance and frequency) may have contributed to the increased P300 amplitude to target vs. non-target stimuli but can not be disentangled (Pritchard, 1981). In addition, as task relevance of a stimulus rather influences the $\mathrm{P} 3 \mathrm{~b}$ and infrequency or deviance the P3a (see e.g., Polich, 2007), this may have led to more comparable sagittal topographies of the P300 in the target and non-target trials.

More important for the aim of the study were the P300 differences between the age groups: in both tasks an age-related reduction of the $\mathrm{P} 3 \mathrm{~b}$ was evident for the target condition at centroparietal and parietal electrode sites. Such an age-related decline of the P3b amplitude during task-relevant processing is well in line with many earlier studies using various paradigms (Friedman et al., 1993). However, the P3b in the easy two-choice task was not generally smaller in amplitude in the middle-aged compared to the young participants because amplitudes were comparable in the non-target condition. In addition, the age-related amplitude reduction of the P300 to targets in the 2-back task, i.e., under high working memory load, was not confined to the P3b. An agerelated amplitude reduction was also evident at fronto-central sites and suggests that also working memory sub-processes may be affected which are reflected by the frontal P3a. This may dismiss the hypothesis of higher compensatory activation in our middleaged group under high working memory load which could be inferred by other studies (e.g., Reuter-Lorenz et al., 2001). Taken together, the pronounced age-related decrease in P300 amplitude under high as compared to low working memory load goes hand in hand with the lower performance of the middle-aged participants especially in the 2-back task.
Crucially, the P300 in the 2-back task showed characteristic topographical differences between the age groups: in the target condition, both groups showed a pronounced P300 at midline and right electrode sites. Specific differences between P300 lateralization and group were, however, found in the non-target condition. Here, the young group also showed the midline and right pronounced P300 topography whereas for the middle-aged participants the P300 was only pronounced at the midline. This difference is surprising as in the present 2-back task, the same sub-processes of working memory are likely to be involved during the processing of target and of non-target stimuli: each letter/stimulus has to be compared with the last but one letter, the content of working memory has to be renewed and the new order has to be encoded and stored until the next stimulus will appear (retrieval, monitoring, updating, storage). Finally, the correct response has to be selected. Therefore, if the P300 may be a reflection of the relevant processes, one may expect to a certain degree comparable topographies between the two trial types. The general amplitude differences may be explainable by the difference in probability of occurrence of the infrequent targets and the frequent non-targets or alternatively may be interpreted as an index of recognition of a critical and behaviorally relevant event. The lack of a midline-right pronounced P300 in the non-target condition only for the middle-aged but not for the young participants may reflect less efficient sub-processes like short-term storage and retrieval which are supported by parietal brain areas (Marshuetz et al., 2006) and/or of the processes which are supported by the frontal lobe (Fletcher and Henson, 2001; Smith et al., 1998). The right lateralized age-related amplitude attenuation was rather general and not confined to the frontal or the parietal sub-component of the P300. The mere consideration of the topographical differences is, in fact, not very informative about the functional processes which may be different in young and middle-aged participants in the non-target condition. Therefore, the confined analysis of the P300 amplitude in non-target trials directly preceding target trials in combination with a source analysis of expected differences may provide additional evidence about the functional meaning of the P300 to non-targets in the 2-back task. The confined analysis revealed two important things: (a) The P300 to non-targets preceding correct targets is significantly attenuated in middle-aged vs. young participants at right lateralized electrodes. (b) The P300 to non-targets is higher before correct targets vs. missed targets only in the young group. This difference was not observed for the middle-aged group.

Interpreting the former result (a), it seems that the increased P300 preceding a correctly detected target indicates a higher activation level of brain areas which support working memory functioning and enhance the probability to detect efficiently the target stimulus in the following trial. This interpretation was underlined by the significant correlation of the P300 and the IE: the higher the amplitude of the P300 at right lateralized electrodes in non-target trials directly before correctly detected targets, the better the general task performance. This correlation did not only emerge in the young but also in the middle-aged group. The group difference of the P300 was further analyzed by sLORETA. This analysis suggests that the young vs. middle-aged group showed higher activation in bilateral medial and right inferior, middle, and superior frontal brain areas as well as in right inferior parietal brain areas in 
non-target trials preceding correctly classified targets. Because the sLORETA analysis is based on the ERPs with limited spatial resolution, the results may be interpreted with caution. Nevertheless, they fit well with the results of other studies which are based on functional neuroimaging, pointing to ventrolateral and dorsolateral frontal brain areas and the inferior parietal gyrus as being important for working memory functioning (e.g., Fletcher and Henson, 2001; Marshuetz et al., 2006) and which may mediate an age-related decline in working memory performance (e.g., Mitchell et al., 2000; Jennings et al., 2006; Mattay et al., 2006; Nyberg et al., 2009).

Therefore, we propose that sub-processes of working memory which are supported by the ventro-lateral frontal cortex (maintenance and rehearsal of information: e.g., Fletcher and Henson, 2001), by the dorsolateral frontal cortex (manipulation of information: e.g., Smith et al., 1998; Fletcher and Henson, 2001) and by parietal brain areas (short-term storage and retrieval: e.g., Marshuetz et al., 2006; Chatham et al., 2011) may be compromised in our middle-aged compared to the young group (e.g., Mitchell et al., 2000; Jennings et al., 2006; Mattay et al., 2006; Nyberg et al., 2009).

Because the present paradigm did not include irrelevant stimuli, our results do not allow to infer that an age-related deficit in filtering or suppressing of irrelevant information does also contribute to the age-related performance decline under high working memory load (see e.g., Gazzaley et al., 2005). Further studies may include irrelevant stimuli or stimulus dimensions within the n-back paradigm in order to examine also this alternative which may not be exclusive but rather complementary in order to explain age-related deficits in working memory.

The latter finding (b) suggests that the P300 has a predictive value for the ongoing action especially in the young participants. In other words, a higher amplitude of the P300 indicates an efficient maintenance of the relevant information, whereas a smaller amplitude indicates a loss of information. The difference in the middle-aged participants was attenuated which may reflect increasing fragility of the matching process. The notion of a predictive value of the P300 regarding performance is also supported by a reliable correlation between the P300 amplitude and IE as an integrative index of overall performance.

The increased amplitude of the P300 in non-target trials preceding correctly classified vs. missed targets in the young group may be explained by higher activation in the right insula and in the posterior cingulum. Although the posterior cingulum has been discussed with the default mode network during resting (van den Heuvel et al., 2008), it has been also connected to consciousness in general (Vogt

\section{REFERENCES}

Babcock, R. L., and Salthouse, T. A. (1990). Effects of increased processing demands on age differences in working memory. Psychol. Aging 5, 421-428.

Baddeley, A. (1992). Working memory. Science 255, 556-559.

Chatham, C. H., Herd, S. A., Brant, A. M., Hazy, T.E., Miyake,A., O'Reilly, R., and Friedman, N.P. (2011). From an executive network to executive control: a computational model of the n-back task. J. Cogn. Neurosci. doi: 10.1162/ jocn_a_00047. [Epub ahead of print].
Dobbs, A. R., and Rule, B. G. (1989). Adult age differences in working memory. Psychol. Aging 4, 500-503.

Esposito, F., Aragri, A., Latorre, V., Popolizio, T., Scarabino, T., Cirillo, S., Marciano, E., Tedeschi, G., and Di Salle, F. (2009). Does the default-mode functional connectivity of the brain correlate with working-memory performances? Arch. Ital. Biol. 147, 11-20.

Fletcher, P. C., and Henson, R. N. (2001). Frontal lobes and human memory: insights from functional neuroimaging. Brain 124, 849-881.

and Laureys, 2005). Assuming, that the higher activation of the posterior cingulum in non-target trials preceding correct targets is related to the default mode network, it is possible that successful matching and manipulation of information in working memory may signal lower cognitive load, and thus, leading to higher activation of the default mode network. In contrast, if, in case of lapses, the processes of matching and manipulation are unsuccessful, this may signal higher cognitive load and the need to be attentive, thus, lowering the activation of the default mode network (see also Esposito et al., 2009). Accordingly, Menon and Uddin (2010) proposed a model of insula function which connects the insula to the detection of salient events and the facilitated access of such stimuli to attention and working memory resources. In return, lapses of performance in young participants which may provoke the missing of upcoming targets were paralleled by lower activation of these brain areas.

\section{SUMMARY AND CONCLUSION}

In summary, the performance of the middle-aged vs. young participants was characterized by a general decline of performance under high working memory load. The electrophysiological data suggest that both, processes which are supported by the frontal lobe and by parietal brain areas are compromised by increasing age, which may explain the age-related reduction of performance in the 2-back task. In contrast, the young participants were able to flexibly tune the relevant processes for task accomplishment under high working memory load. Lapses of performance in the young group were paralleled by lower activation of brain areas which support attentional switching (Menon and Uddin, 2010) and consciousness (Vogt and Laureys, 2005). In contrast, the middle-aged group showed the same neuronal pattern regardless of whether a subsequent target will be detected or misses. The stable memory trace in the young compared to the middle-aged participants was reflected by a specific activation enhancement in brain areas which orchestrate maintenance, update, storage, and retrieval of information in working memory even before the critical event occurred.

\section{ACKNOWLEDGMENTS}

We thank Gabrielle Freude and Sergei Schapkin (BAuA, Berlin) for the supervision of the study, Claudia Wipking and Ines Mombrei (IfADo) for assistance in running the experiments Udo Erdmann for programming and Ludger Blanke for his technical expertise and support. The study was supported by the INQA-Initiative for New Quality of Work and founded by the Federal Ministry of Labour and Social Affairs (BMAS).

Friedman, D., Kazmerski, V., and Fabiani, M. (1997).An overview of age-related changes in the scalp distribution of P3b. Electroencephalogr. Clin. Neuophysiol. 104, 498-513.

Friedman, D., Simpson, G., and Hamberger, M. (1993). Age-related changes in scalp topography to novel and target stimuli. Psychophysiology 30, 383-396.

Gazzaley, A., Cooney, J. W., Rissman, J., and D'Esposito, M. (2005). TopDown suppression deficit underlies working memory impairment in normal aging. Nat. Neurosci. 8, 1298-1300.

Gratton, G., Coles, M. G. H., and Donchin, E. (1983). A new method for off-line removal of ocular artifact. Electroencephalogr. Clin. Neuophysiol. 55, 468-484.

Hasher, L., and Zacks, R. T. (1988). "Working memory, comprehension, and aging: a review and a new view," in The Psychology of Learning and Motivation, Vol. 22, ed. G. H. Bower (San Diego: Academic Press), 193-225. 
Holmes, A. P., Blair, R. C., Watson, J. D., and Ford, I. (1996). Nonparametric analysis of statistic images from functional mapping experiments. J. Cereb. Blood Flow Metab. 16, 7-22.

Jennings, J. R., van der Veen, F. M., and Meltzer, C. C. (2006). Verbal and spatial working memory in older individuals: a positron emission tomography study. Brain Res. 1092, 177-189.

Koenigs, M., Barbey, A. K., Postle, B. R., and Grafman, J. (2009). Superior parietal cortex is critical for the manipulation of information in working memory. J. Neurosci. 29, 14980-14986.

Li, S. C., Lindenberger, U., and Sikstrom, S. (2001). Aging cognition: from neuromodulation to representation. Trends Cogn. Sci. 5, 479-486.

Marshuetz, C., Reuter-Lorenz, P.A., Smith, E. E., Jonides, J., and Noll, D. C. (2006). Working memory for order and the parietal cortex: an event-related functional magnetic resonance imaging study. Neuroscience 139, 311-316.

Mattay, V. S., Fera, F., Tessitore, A., Hariri, A. R., Berman, K. F., Das, S., MeyerLindenberg, A., Goldberg, T. E., Callicott, J. H., and Weinberger, D. R. (2006). Neurophysiological correlates of age-related changes in working memory capacity. Neurosci. Lett. 392, 32-37.

Menon, V., and Uddin, L. Q. (2010). Saliency, switching, attention and control: a network model of insula function. Brain Struct. Funct. 214, 655-667.

Missonnier, P., Gold, G., Leonards, U., Costa-Fazio, L., Michel, J. P., Ibanez, V., and Giannakopoulos, P. (2004). Aging and working memory: early deficits in EEG activation of posterior cortical areas. J. Neural Transm. 111, 1141-1154.

Missonnier,P.,Leonards, U., Gold, G.,Palix, J., Ibanez, V., and Giannakopoulos, P. (2003). A new electrophysiological index for working memory load in humans. Neuroreport 14, 1451-1455.

Mitchell, K. J., Johnson, M. K., Raye, C. L., Mather, M., and D'Esposito, M. (2000). Aging and reflective processes of working memory: binding and test load deficits. Psychol. Aging 15, 527-541.

Nyberg, L., Dahlin, E., Stigsdotter Neely, A., and Backman, L. (2009). Neural correlates of variable working memory load across adult age and skill: dissociative patterns within the frontoparietal network. Scand. J. Psychol. 50, 41-46.

Pascual-Marqui, R. D. (2002). Standardized low-resolution brain electromagnetic tomography (sLORETA): technical details. Methods Find Exp. Clin. Pharmacol. 24(Suppl. D), 5-12.

Pivik, R. T., Broughton, R. J., Coppola, R., Davidson, R. J., Fox, N., and Nuwer, M. R. (1993). Guidelines for the recording and quantitative analysis of electroencephalographic activity in research contexts. Psychophysiology 30, 547-558.

Polich, J. (2007). Updating P300: an integrative theory of $\mathrm{P} 3 \mathrm{a}$ and $\mathrm{P} 3 \mathrm{~b}$. Clin. Neurophysiol. 118, 2128-2148.

Pritchard,W.S. (1981). Psychophysiology of P300. Psychol. Bull. 89, 506-540.

Raz, N., Gunning-Dixon, F. M., Head, D., Dupuis, J. H., and Acker, J. D. (1998). Neuroanatomical correlates of cognitive aging: evidence from structural magnetic resonance imaging. Neuropsychology 12, 95-114.
Reuter-Lorenz, P. A., Jonides, J., Smith, E. E., Hartley, A., Miller, A., Marshuetz, C., and Koeppe, R. A. (2000). Age differences in the frontal lateralization of verbal and spatial working memory revealed by PET. J. Cogn. Neurosci. 12, 174-187.

Reuter-Lorenz, P. A., Marshuetz, C., Jonides, J., Smith, E. E., Hartley, A., and Koeppe, R. (2001). Neurocognitive ageing of storage and executive processes. Eur. J. Cogn. Psychol. 13, 257-278.

Salat, D. H., Kaye, J. A., and Janowsky, J. S. (1999). Prefrontal gray and white matter volumes in healthy aging and Alzheimer disease. Arch. Neurol. 56, 338-344.

Salthouse, T. A. (2000). Aging and measures of processing speed. Biol. Psychol. $54,35-54$

Schmiedek, F., Li, S. C., and Lindenberger, U. (2009). Interference and facilitation in spatial working memory: age-associated differences in lure effects in the n-back paradigm. Psychol. Aging 24, 203-210.

Smith, E. E., Jonides, J., Marshuetz, C., and Koeppe, R. A. (1998). Components of verbal working memory: evidence from neuroimaging. Proc. Natl. Acad. Sci. U.S.A. 95, 876-882.

Sutton, S., Braren, M., Zubin, J., and John, E. R. (1965). Evoked-potential correlates of stimulus uncertainty. Science 150, 1187-1188.

Townsend, J. T., and Ashby, F. G. (1983). Stochastic Modeling of Elementary Psychological Processes. Cambridge: Cambridge University Press.

van den Heuvel, M., Mandl, R., Luigjes, J., and Hulshoff Pol, H. (2008). Microstructural organization of the cingulum tract and the level of default mode functional connectivity. $J$. Neurosci. 28, 10844-10851.

Vogt, B. A., and Laureys, S. (2005). Posterior cingulate, precuneal and retrosplenial cortices: cytology and components of the neural network correlates of consciousness. Prog. Brain Res. 150, 205-217.

West, R. (1996). An application of prefrontal cortex function theory of cognitive aging. Psychol. Bull. 120, 272-292.

Wild-Wall, N., and Falkenstein, M. (2010). Age-dependent impairment of auditory processing under spatially focused and divided attention: an electrophysiological study. Biol. Psychol. 83, 27-36.

Conflict of Interest Statement: The authors declare that the research was conducted in the absence of any commercial or financial relationships that could be construed as a potential conflict of interest.

Received:09 June 2011; paperpending published: 24 June 2011; accepted: 22 July 2011; published online: 30 August 2011.

Citation: Wild-Wall N, Falkenstein M and Gajewski PD (2011) Age-related differences in working memory performance in a 2-back task. Front. Psychology 2:186. doi: 10.3389/fpsyg.2011.00186

This article was submitted to Frontiers in Cognition, a specialty of Frontiers in Psychology.

Copyright (C) 2011 Wild-Wall, Falkenstein and Gajewski. This is an open-access article subject to a non-exclusive license between the authors and Frontiers Media SA, which permits use, distribution and reproduction in other forums, provided the original authors and source are credited and other Frontiers conditions are complied with. 